\title{
Neuropsychiatric Challenges in the Era of Direct-Acting Antivirals: A Case-Report
}

\section{Peixoto I* \\ Centro Hospitalar Lisboa Central, Portugal}

*Corresponding author: Ivo Peixoto, Centro Hospitalar Lisboa Central, Rua Jacinta Marto Lisboa, Portugal, Tel: 00351968206163; Email: ivopintopeixoto@gmail.com

\section{Case Report}

Volume 2 Issue 1

Received Date: May 03, 2018

Published Date: May 22, 2018

DOI: $10.23880 / \mathrm{mhrij}-16000118$

\section{Abstract}

In the advent of new all-pill treatments for chronic hepatitis $C$ virus infection, there is a myriad of clinical challenges in the management of the psychiatric implications and side effects of such therapies, especially in the patient with major psychiatric disorder, which is a very frequent comorbidity. We describe, for the first time, the clinical case of a patient diagnosed with schizophrenia manifesting clinical decompensation associated with the start of a sofosbuvir-containing treatment regimen. We aim to illustrate and provide a discussion about the clinical challenges and psychiatric management issues posed by a patient with a major mental disorder under a sofosbuvir-containing antiviral regimen for HCV infection.

Keywords: Psychiatry; Mental Disorders; Direct-acting antivirals; Sofosbuvir

\section{Introduction}

With the proliferation of new treatments for chronic hepatitis $\mathrm{C}$ virus (HCV), we may now be in the era of allpill direct-acting antiviral regimens. This has potentially relevant management implications for psychiatry, since people with severe mental illness are a high-prevalence population of HCV infection, as recently reviewed [1]. In addition, psychiatric comorbidity in HCV-infected patients seems to be the rule rather than the exception [2]. As HCV therapy moves forward towards interferon-alpha-free regimens, more patients with severe mental illness and substance use diagnoses will likely receive HCV treatment. Although these regimens show significantly better safety and psychiatric side effect profiles, neuropsychiatric risks of HCV therapy continue to be a significant concern $[3,4]$. We describe here, for the first time, a patient diagnosed with chronic hepatitis $\mathrm{C}$ virus infection and paranoid schizophrenia in full clinical remission, who developed a relapse of psychotic symptomatology together with sleep and behavioral alterations after initiation of a sofosbuvir-containing antiHCV treatment regimen.

\section{Case Report}

Mr. C.H., a 52-year-old man, was admitted in a psychiatric inpatient unit presenting delusional beliefs and auditory-verbal hallucinations for several weeks. The patient had a 30 year history of HCV chronic infection and schizophrenia with a chronic relapsing course and had been treated with different psychotropic agents in multiple psychiatric structures with difficult symptom stabilization. For the previous 3 years, he remained however, clinically asymptomatic under haloperidol decanoate $100 \mathrm{mg}$ twice per month, olanzapine $30 \mathrm{mg}$ /day, 


\section{Mental Health \& Human Resilience International Journal}

ciamemazine $200 \mathrm{mg} /$ day, and benzodiazepines for sleep problems.

He had initiated HCV therapy with ribavirin plus sofosbuvir three months before admission, and started to exhibit complaints of total insomnia, growing irritability and later development of relapsing psychotic symptoms with auditory-verbal hallucinations and delusional beliefs with increasing dynamism and behavioral and functional impairment.

Upon admission, hemogram, basic analytic chemistry and urianalysis, showed no alterations. Since he was on the $14^{\text {th }}$ week of rivabirin plus sofosbuvir regimen and attained negative viral loads on the second week of treatment we opted for the discontinuation of the antiHCV regimen. No alterations were made in the psychotropic prescription. In the following days after admission, the patient showed a favorable clinical course, with remission of psychotic symptoms and resolution of sleep problems, and was discharged 3 weeks later. He remained asymptomatic at the 2-months follow-up visit.

\section{Discussion and Conclusions}

This is, to our knowledge, the first clinical case report published so far on the neuropsychiatric implications of a sofosbuvir-containing treatment regimen in a patient with major psychiatric illness.
The recent arrival of direct-acting antivirals in the treatment of chronic HCV infection increase the likelihood of cure with a shorter duration of treatment and better side effect profile, when compared with previous HCV medications [4]. Mr. C. is among the $50 \%$ of patients with HCV infection who also have a history of psychiatric illness [5].

In the clinical case presented, it is noteworthy to mention the possible mechanisms for causation of psychiatric illness decompensation: a) direct-acting antiviral psychiatric side-effects; b) direct-acting antivirals' potential interaction profile with psychotropic medications altering exposures of either/both drug classes; c) direct antivirals may alter neurobiology with possible implications on psychiatric illness pathophysiology and clinical course.

According to published data so far, sofosbuvircontaining regimens for HCV treatment do not appear to confer additional neuropsychiatric risks to patients and are far more readily tolerated than the previous regimens [6]. However, data collected from clinical trials still report appreciable rates of psychiatric symptomatology, particularly fatigue $(23-47 \%)$, insomnia $(11-29 \%)$ and irritability (5-15\%) (Table 1). The rates of such manifestations in people with major psychiatric illness remains largely unknown.

\begin{tabular}{|c|c|c|c|c|}
\hline \multicolumn{5}{|c|}{ Sofosbuvir Trials } \\
\hline \multirow{2}{*}{ Side Effect } & FISSION & FUSION & POSITRON & VALENCE \\
\hline & (Lawitz et al. 2013) [7] & (Jacobson et al. 2013) [8] & (Jacobson et al. 2013) [8] & (Zeuzem et al. 2014) [9] \\
\hline Fatigue & $36 \%$ & $44 \%$ & $47 \%$ & $23-30 \%$ \\
\hline Insomnia & $12 \%$ & $19 \%$ & $29 \%$ & $11-16 \%$ \\
\hline Irritability & $10 \%$ & $9 \%$ & $15 \%$ & $5-10 \%$ \\
\hline
\end{tabular}

Table 1: Psychiatric adverse effects in Sofosbuvir trials.

Furthermore, the use of direct-acting antivirals requires careful recognition of potential drug-drug interactions with psychotropic agents and an analysis of whether psychotropic regimens should be changed due to significant interaction risks. The potential for pharmacokinetic or pharmacodynamic interactions with psychotropic agents may also exacerbate side effects and interfere with direct-acting antiviral compliance, thus reducing HCV treatment efficacy.

Studies specifically examining neuropsychiatric complications of treatment with direct-acting antivirals for hepatitis $\mathrm{C}$ in patients with major psychiatric illnesses, as well as clinical recommendations regarding these new challenges, are still lacking. Further investigation on the psychiatric clinical monitoring of such patients will likely advance our knowledge of symptomatic and drug interaction profiles.

The psychiatric approach of patients infected with HCV will hopefully move from tackling frequent and challenging neuropsychiatric symptomatology of previous treatment regimens towards the improvement of engagement and treatment adherence, management of unique drug-drug interactions and ongoing support during and after HCV treatment. However, we still need 


\section{Mental Health \& Human Resilience International Journal}

further evidence to increase our understanding of directacting antiviral related psychiatric complications, and to clarify the clinical translation of evidence published so far. This will be essential to redefine the role of psychiatric care in the evolving area of HCV treatment.

\section{References}

1. Hughes E, Bassi S, Gilbody S, Bland M, Martin F (2016) Prevalence of HIV, hepatitis B, and hepatitis C in people with severe mental illness: a systematic review and meta-analysis. The Lancet Psychiatry 3(1): 40-48.

2. Rifai MA, Indest D, Loftis J, Hauser P (2006) Psychiatric management of the hepatitis $C$ patient. Curr Treat Options Gastro 9(6): 508-519.

3. Sockalingam S, Tseng A, Giguere P, Wong D (2013) Psychiatric treatment considerations with direct acting antivirals in hepatitis C. BMC Gastroenterology 13: $1-10$.

4. Sockalingam S, Sheehan K, Feld JJ, Shah H (2015) Psychiatric Care During Hepatitis C Treatment: The Changing Role of Psychiatrists in the Era of Direct-
Acting Antivirals. American Journal of Psychiatry 172(6): 512-516.

5. Rifai MA, Gleason OC, Sabouni D (2010) Psychiatric Care of the Patient With Hepatitis C: A Review of the Literature. Primary Care Companion J Clin Psychiatry 12(6).

6. Younossi Z, Henry L (2015) Systematic review: patient-reported outcomes in chronic hepatitis C--the impact of liver disease and new treatment regimens. Aliment Pharmacol Ther 41(6): 497-520.

7. Lawitz E, Mangia A, Wyles D, Rodriguez-Torres M, Hassanein T, et al. (2013) Sofosbuvir for Previously Untreated Chronic Hepatitis C Infection. N Engl J Med 368(20): 1878-1887.

8. Jacobson IM, Gordon SC, Kowdley KV, Yoshida EM, Rodriguez-Torres M, et al. (2013) Sofosbuvir for Hepatitis C Genotype 2 or 3 in Patients without Treatment Options. New England Journal of Medicine 368(20): 1867-1877.

9. Zeuzem S, Dusheiko GM, Salupere R, Mangia A, Flisiak $\mathrm{R}$, et al. (2014) Sofosbuvir and Ribavirin in HCV Genotypes 2 and 3. N Eng J Med 370(21): 1993-2001.

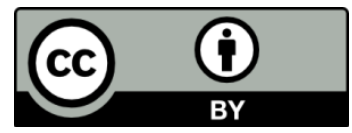

